\title{
Real-time loop-mediated isothermal amplification for rapid detection of Enterocytozoon hepatopenaei
}

\author{
Shao xin Cai ${ }^{1,2}$, Fande Kong ${ }^{2}$, ShuFei XU ${ }^{\text {Corresp., }}{ }^{2}$, Cuiluan YAO ${ }^{\text {Corresp. } 1}$ \\ ${ }^{1}$ Fisheries College, Jimei University, Xiamen, China \\ 2 State Key Laboratory for Crustaceans Quarantine, Xiamen Customs, Xiamen, China \\ Corresponding Authors: ShuFei XU, Cuiluan YAO \\ Email address: xusf@xmciq.gov.cn, clyao@jmu.edu.cn
}

Background. Enterocytozoon hepatopenaei ( EHP ) is a newly emerged microsporidian parasite that causes retarded shrimp growth in many countries. But there are no effective approaches to control this disease to date. The EHP could be an immune risk factor for increased dissemination of other diseases. Further, EHP infection involves the absence of obvious clinical signs and it is difficult to identify the pathogen through visual examination, increasing the risk of disease dissemination. It is urgent and necessary to develop a specific, rapid and sensitive EHP-infected shrimp diagnostic method to detect this parasite. In the present study, we developed and evaluated a rapid real-time Loop-Mediated Isothermal Amplification (real-time LAMP) for detection of EHP.

Methods. A rapid and efficient real-time LAMP method for the detection of EHP has been developed. Newly emerged EHP pathogens in China were collected and used as the sample, and three sets of specificity and sensitivity primers were designed. Three other aquatic pathogens were used as templates to test the specificity of the real-time LAMP assay. Also, we compared the real-time LAMP with the conventional LAMP by the serial dilutions of EHP DNA and their amplification curves. Application of realtime LAMP was carryed out with clinical samples.

Results. Positive products were amplified only from EHP, but not from other tested species, EHP was detected from the clinical samples, suggesting a high specificity of this method. The final results of this assay were available within less than $45 \mathrm{~min}$, and the initial amplification curve was observed at about six min. We found that the amplification with an exponential of 6-fold dilutions of EHP DNA demonstrated a specific positive signal by the real-time LAMP, but not for the LAMP amplicons from the visual inspection. The real-time LAMP amplification curves demonstrated a higher slope than the conventional LAMP.

Discussion. In this study, pathogen virulence impacts have been increased in aquaculture and continuous observation was predominantly focused on EHP. The present study confirmed that the realtime LAMP assay is a promising and convenient method for the rapid identification of EHP in less time and cost. Its application greatly aids in the detection, surveillance, and prevention of EHP. 
1

2 Real-Time Loop-Mediated Isothermal Amplification for

3 Rapid Detection of Enterocytozoon hepatopenaei

4 Shaoxin $\mathrm{CAI}^{1,2}$, Fande KONG ${ }^{2}$, Shufei $\mathrm{XU}^{2}$, Cuiluan $\mathrm{YAO}^{1}$,

5 1. Fisheries College, Jimei University, Xiamen, Fujian, China

6

7

8

9

10

11

12

13

14

15

16

17

18

19

20

21

22

23

24

25

26

27

28

29
Corresponding Author:

1.Cuiluan YAO

Fisheries College, Jimei University, Xiamen, Fujian, 361021, China

Email address: clyao@jmu.edu.cn

2.Shufei XU

State Key Laboratory for Crustaceans Quarantine, Xiamen Customs, Xiamen, Fujian, China

Email address: xusf@xmciq.gov.cn 


\section{Abstract:}

Background. Enterocytozoon hepatopenaei (EHP) is a newly emerged microsporidian parasite that causes retarded shrimp growth in many countries. But there are no effective approaches to control this disease to date. The EHP could be an immune risk factor for increased dissemination of other diseases. Further, EHP infection involves the absence of obvious clinical signs and it is difficult to identify the pathogen through visual examination, increasing the risk of disease dissemination. It is urgent and necessary to develop a specific, rapid and sensitive EHPinfected shrimp diagnostic method to detect this parasite. In the present study, we developed and evaluated a rapid real-time Loop-Mediated Isothermal Amplification (real-time LAMP) for detection of EHP.

Methods. A rapid and efficient real-time LAMP method for the detection of EHP has been developed. Newly emerged EHP pathogens in China were collected and used as the sample, and three sets of specificity and sensitivity primers were designed. Three other aquatic pathogens were used as templates to test the specificity of the real-time LAMP assay. Also, we compared the real-time LAMP with the conventional LAMP by the serial dilutions of EHP DNA and their amplification curves. Application of real-time LAMP was carried out with clinical samples.

Results. Positive products were amplified only from EHP, but not from other tested species, EHP was detected from the clinical samples, suggesting a high specificity of this method. The final results of this assay were available within less than $45 \mathrm{~min}$, and the initial amplification curve was observed at about six min. We found that the amplification with an exponential of 6fold dilutions of EHP DNA demonstrated a specific positive signal by the real-time LAMP, but not for the LAMP amplicons from the visual inspection. The real-time LAMP amplification curves demonstrated a higher slope than the conventional LAMP. 
53 Discussion. In this study, pathogen virulence impacts have been increased in aquaculture 54 and continuous observation was predominantly focused on EHP. The present study confirmed

55 that the real-time LAMP assay is a promising and convenient method for the rapid identification

56 of EHP in less time and cost. Its application greatly aids in the detection, surveillance,

57 andprevention of EHP.

58 Keywords: shrimp disease, Enterocytozoon hepatopenaei, diagnosis, real-time Loop-

59 mediated isothermal amplification, rapid detection

60

61

62

63

64

65

66

67

68

69

70

71

72

73

74

75 


\section{Introduction}

Enterocytozoon hepatopenaei (EHP) is the microsporidian parasite that causes

hepatopancreatic microsporidiosis (HPM) in shrimp (Chayaburakul et al., 2004; Tangprasittipap et al., 2013). Moreover, EHP is an emerging pathogen that affects the cultured shrimp Penaeus vannamei in many countries, such as Vietnam, Thailand, Brunei, Malaysia, Indonesia, China and Venezuela (Chayaburakul et al., 2004; Liu et al., 2017; Rajendran et al., 2016; Tang et al., 2016; Tang et al., 2015; Tangprasittipap et al., 2013; Thi Ha et al., 2010), and is imposing a continuous threat to shrimp farming industries in future. The shrimp with EHP could be an immune risk factor, causing an increased susceptibility to pathogens of both acute hepatopancreatic necrosis disease and septic hepatopancreatic necrosis (Aranguren et al., 2017).

Enterocytozoon hepatopenaei is an intracellular parasite that replicates within the cytoplasmic area of the tubule epithelial cells in the hepatopancreas, which was considered to be a new species, E. hepatopenaei, within the genus Enterocytozoon (Tourtip et al. 2009). Further, EHP infection involves the absence of obvious clinical signs and it is difficult to identify the pathogen through visual examination, increasing the risk of disease dissemination. To control EHP, the EHP-infected broodstock from the cultivation system is excluded. However, it is difficult to perceive EHP due to the absence of obvious clinical symptoms when the shrimp was infected. Hence, it is urgent and necessary to develop a specific, rapid and sensitive EHPinfected shrimp diagnostic method to detect this parasite.

It is well known that modern molecular biology detection techniques play an important role in pathogen detection, and there have been major improved techniques, such as PCR assay, in situ hybridization, and real-time PCR put forward in recent years (Cai et al., 2012). But due to expensive equipment, and reagents limited their broader application. Also, histological 
99

100

101

102

103

104

105

106

107

108

109

110

111

112

113

114

115

116

117

118

119

120

121

observations were limited due to the individual size of EHP, and it remained difficult to detect some small individual (Tourtip et al. 2009). While in situ hybridization has a low sensitivity and may lead to few false-positive results. Consequently, a rapid detection method for EHP is necessary for suitable handling, prevention of spreading and reducing the risk factors caused by this pathogen.

In order to develop a faster, more reliable and more cost-effective assays, we applied a loopmediated nucleic acid isothermal amplification (LAMP) to develop a real-time loop-mediated isothermal amplification (real-time LAMP) for detecting EHP. The real-time LAMP assay is a more sensitivity and accurate diagnostic method, which enables the detection of low-level infections and infection in the host. This assay remained a critical tool to detect EHP in shrimps.

\section{Materials and methods}

\section{Animal samples}

Four EHP positive shrimp samples are naturally infected were collected from the shrimp ponds in Zhanjiang, Guangdong, China in February 2017. All the positive samples were confirmed by the state key laboratory for crustacean quarantine. Clinical samples were collected from the shrimp ponds in Zhanjiang, Guangdong, China. Then, the dissected hepatopancreatic tissues of the shrimp were taken and preserved separately using 95\% ethanol with more than three times the volume. The White spot syndrome virus (WSSV), Macrobrachium rosenbergii Nodavirus (MrNV), and Infectious hypodermal and hematopoietic necrosis virus (IHHN) used in the present study was provided by the research teams at state key laboratory for crustaceans quarantine.

\section{DNA extraction and template preparation}

The hepatopancreatic tissues were rinsed under sterile water to remove ethanol. This was 
122 followed by mixing of 50mg 1g of shrimp hepatopancreatic tissues with two times the volume 123 of lysis buffer (100 mM pH 8.0 Tris-HCl, $1 \mathrm{mM} \mathrm{pH} \mathrm{8.0} \mathrm{EDTA,} \mathrm{1 \%} \mathrm{SDS} \mathrm{and} \mathrm{sterile} \mathrm{water)} \mathrm{at}$ 124 room temperature for $5 \mathrm{~min}$, and then lysed at $95^{\circ} \mathrm{C}$ for $5 \mathrm{~min}$. The mixture was transferred to the 125 DNeasy Mini spin column and centrifuged for $1 \mathrm{~min}$. Serial dilutions of EHP DNA ( $10^{0}$ to $\left.10^{-6}\right)$

126

were prepared using Tris buffer (TE, $\mathrm{pH} 8.0$ ), and then stored at $20^{\circ} \mathrm{C}$ until further use.

\section{Oligonucleotides for real-time LAMP}

The real-time LAMP primers targeting EHP were selected from the established methods for this study (Table1). The real-time LAMP primers (EHP-OF, EHP-OB, EHP-IF, EHP-IB, EHPLF, EHP-LB) and LAMP primers were designed using Primer Explorer ver. 4

(https://primerexplorer.jp/elamp4.0.0/index.html). These primers were synthesized by Sangon Biotech Co., Ltd (Shanghai, China).

\section{Real-time LAMP reaction}

The real-time LAMP reaction was performed in a total volume of $25 \mu 1$, including $0.5 \mu \mathrm{l}(8 \mathrm{U})$ of Bst DNA Polymerase Large Fragment (NEB, Beijing, China), $4 \mu \mathrm{M}$ of SYTO-9 (Thermo Fisher Scientific, Shanghai, China), $1 \mu$ EHP-OF \& EHP-OB (0.2 $\mu \mathrm{mol} / \mathrm{L}), 1 \mu 1$ EHP-IF \& EHP-IB (1.6 $\mu \mathrm{mol} / \mathrm{L}), 1 \mu \mathrm{l}$ EHP-LF \& EHP-LB $(0.8 \mu \mathrm{mol} / \mathrm{L}), 1 \mu \mathrm{l}$ of EHP template DNA, and $13 \mu \mathrm{l}$ of real-time LAMP buffer. The real-time LAMP buffer contains $20 \mathrm{mM}$ Tris-HCl, $10 \mathrm{mM}$ $\mathrm{KCl}, 10 \mathrm{mM}\left(\mathrm{NH}_{4}\right)_{2} \mathrm{SO}_{4}, 2 \mathrm{mM} \mathrm{MgSO}_{4}, 0.1 \%$ of Triton X-100, $1.4 \mathrm{mM}$ dNTPs (TaKaRa, Dalian, China) each, and sterile water was used to make up to $25 \mu$. The fluorescent signals were observed automatically by StepOne Real-Time PCR System (ABI, Shanghai, China).

\section{LAMP reaction}

The LAMP reaction was performed in a total of $25 \mu$ volume, including $0.5 \mu 1(8 \mathrm{U})$ of Bst DNA Polymerase Large Fragment (NEB, Beijing, China), $4 \mu \mathrm{M}$ of SYTO-9 (Thermo Fisher 
145 Scientific, Beijing, China), $1 \mu \mathrm{l}, 12.5 \mu \mathrm{l}$ of LAMP buffer (containing $20 \mathrm{mM}$ Tris-HCl, $10 \mathrm{mM}$

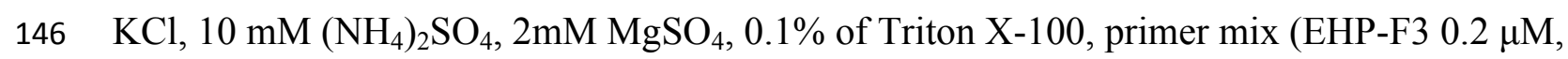

147 EHP-B3 0.2 $\mu \mathrm{M}$, EHP-FIP 1.6 $\mu \mathrm{M}$, EHP-BIP $1.6 \mu \mathrm{M}), 2 \mu 1$ EHP template DNA, $1.4 \mathrm{mM}$ dNTPs

148 each), and sterile water was used to make up to $25 \mu 1$. The reaction was carried out at $60^{\circ} \mathrm{C}$ for

$149 \quad 45-90 \mathrm{~min}$.

150 Specificity of real-time LAMP detection

151 To determine the specificity, the established real-time LAMP method was carried out using 152 different sources of DNA isolated from WSSV, MrNV, IHHN and with EHP template DNA, $153 \mathrm{ddH}_{2} \mathrm{O}$ was used as a negative control. Also, clinical samples were diagnosed with the 154 established real-time LAMP method, the EHP DNA was used as a positive sample, and $\mathrm{ddH}_{2} \mathrm{O}$ 155 was used as a negative control.

\section{The sensitivity of real-time LAMP}

The sensitivity of real-time LAMP procedure developed was compared with LAMP, and the

primers used in the real-time LAMP and LAMP were listed in Table 1. Serial dilutions of EHP

DNA $\left(10^{0}\right.$ to $\left.10^{-6}\right)$ were used to be a positive sample, and $\mathrm{ddH}_{2} \mathrm{O}$ was used as a negative control. The real-time LAMP the fluorescence signal date was obtained from StepOne Real-Time PCR System.

\section{Comparison with the conventional LAMP}

The EHP DNA was used as the template, and $\mathrm{ddH}_{2} \mathrm{O}$ was used as a negative control. The primers used in the real-time LAMP and LAMP were listed in Table 1. The reactions were carried out under the conditions mentioned above. The real-time LAMP was compared with the conventional LAMP by their amplification curves. The fluorescent signals were observed automatically by StepOne Real-Time PCR System. 
168

169

170

171

172

173

174

\section{Results}

\section{The established real-time LAMP primers are tested}

The real-time LAMP was performed with four different EHP samples and $\mathrm{ddH}_{2} \mathrm{O}$ as a control template to test the set of primers. The S type curve demonstrated a positive result, while the smooth straight line a negative result. From the appearance of the amplification curves, the set of primers demonstrated increased fluorescent signals without any amplification peak in the negative control (Fig. 1).

\section{The established real-time LAMP shows high specificity}

To test the specificity of real-time LAMP assay, three other aquatic pathogens were used as templates. The cycle number at which the amplified products surpassed the threshold of detection was observed as the positive fluorescent signal. Along with EHP DNA, various pathogens including WSSV, MrNV, and IHHN have been tested. Positive products were amplified only from EHP, and the curves of the other three tested species showed straight lines, suggesting no amplification (Fig. 2). Also, EHP was detected in clinical samples (data no-show). The specificity test of real-time LAMP revealed that the assay developed showed no crossreactivity with other aquatic pathogens, indicating that the method was highly specific.

\section{The established real-time LAMP is highly sensitive}

Sensitivity analysis was performed using a 10-fold serial dilution set of template DNA of the above mentioned EHP. Serial 10 -fold dilutions ranging from $10^{0}$ times to $10^{-6}$ times of EHP positive sample DNA were tested by real-time LAMP and LAMP. We found that the amplification with an exponential of 6-fold dilutions of EHP DNA demonstrated a specific amplification curve by the real-time LAMP (Fig. 3). From the visual inspection of the LAMP amplicons, the turbidity change of the tube was clearly observed during amplification with an 
191

192

193

194

195

196

197

198

199

200

201

202

203

204

205

206

207

208

209

210

211

212 213

exponential of 5-fold dilutions of EHP DNA, but not for the exponential of 6-fold dilutions of EHP DNA (Fig.4). This indicated that the sensitivity of the real-time LAMP assay was higher than the LAMP.

\section{The established real-time LAMP has high efficiency}

The real-time LAMP has added a pair of loop-primer compared to the conventional LAMP.

The efficiency was compared with the LAMP using their amplification curves. The cycle number at which the amplified products surpassed the threshold of detection could be observed as the positive fluorescent signal. As shown in the figure, real-time LAMP amplification curves demonstrated a higher slope than the conventional LAMP. It took more than 60 cycles for the LAMP to reach the amplification plot, while 14 cycles of amplification for the real-time LAMP to reach the same level (Fig. 5). These results revealed that the real-time LAMP demonstrated more amplification efficiency than the conventional LAMP.

\section{Discussion}

Enterocytozoon hepatopenaei is a newly emerged microsporidian parasite that causes retarded shrimp growth in cultures of many shrimp farming countries, but no effective approaches to control this disease has been developed to date. The pathogens of EHP are hard to eliminate. Complete inhibition of the activity of EHP spores is demonstrated either by freezing the spores at $-20^{\circ} \mathrm{C}$ for at least $2 \mathrm{~h}$ or by treating them with chemicals (Aldama-Cano et al., 2018). It is difficult to distinguish the EHP infected ones with normal ones, and many molecular detection methods have been developed to detect the pathogens.

Recently, many works have been done to detect EHP-infected shrimp, which included histological observation, PCR assay and in situ hybridization (Tangprasittipap et al. 2013), realtime PCR (Liu et al. 2017a). These techniques require complicated equipment such as 
214 thermocycler, electrophoresis set, gel documentation system and trained personnel, and could not

215 be used for on-site detection.

216 The LAMP technique is a rapid, sensitive and specific detection method for the pathogen

217 (Jelocnik et al. 2017), and is also applied to detect the parasites, such as toxoplasmosis (Lau et al.,

218 2010). Therefore, the efficiency of the LAMP is more sensitive than normal PCR. The

219 amplification products of LAMP assays are visually detected by observation (Goo et al. 2016),

220 but the results were always affected by distinctiveness, the results are obtained only after the

221 reaction has been finished, and it cannot distinguish high concentration or low concentration

222 DNA sample.

The real-time LAMP is a new DNA amplification technique. The real-time LAMP was more

224

225

226

227

228

229

230

231

232

233

234

235

236

sensitive than the nested PCR (Sritunyalucksana et al., 2006). Compared to the LAMP, the real-

time LAMP has added a set of loop primers, which increased the efficiency of amplification and

made the detection more rapidly. The positive results were seen as a curve through the screen

directly (Karthikeyan et al., 2017). Low-level infections of DNA pathogens can also be detected

(Mori et al., 2004; Notomi et al., 2000).

The target sequence can be amplified under isothermal conditions with high efficiency, rapidity, and specificity. Positive products were amplified only from EHP, but not from any other tested species. Serial dilution test showed that the real-time LAMP was more sensitive than the LAMP. Because of higher amplification efficiency, the real-time LAMP can save almost half the time than the LAMP. The use of specific loop primers for detecting EHP can reduce the necessary amplification time to less than $45 \mathrm{~min}$, the initial amplification curve was observed from about six min.

In addition, the assay is easy to operate and determine, performed under isothermal 
237 conditions, and can be used by people without any operation experience. The tube lid need not be 238 opened to eliminate the aerosol pollution for excluding the false positive results. While the

239 LAMP product was detected by white precipitate or color change, which is not easy to detect

240 (Deb et al. 2016). It can greatly improve the detection sensitivity and specificity.

241

242

243

244

245

246

247

248

249

250

251

252

253

254

255

256

257

258

259

\section{4}

\section{7}

\section{Conclusions}

In this study, pathogen virulence impacts have been increased in aquaculture and continuous observation was predominantly focused on EHP. The present study confirmed that the real-time LAMP assay is a promising and convenient method for the rapid identification of EHP in less time and cost. Its application greatly aids in the detection, surveillance, and prevention of EHP.

\section{Acknowledgments}

We thank associate Prof. Ming-sheng CAI for the helpful advice assays. This study was supported by the Science and Technology Program of Jimei (20172C01).

9

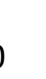

1

2

3

5

56


Fig.1, Primers tested for real-time LAMP

263

264

265

266

267

268

269

270

271

272

273

274

275

276

277

278

279

280

281

282
The amplification curve graph was plotted automatically by StepOne Real-Time PCR System.

$\Delta \mathrm{Rn}$ is the fluorescence unit minus the baseline, the graph depicts the $\Delta \mathrm{Rn}$ on the $\mathrm{Y}$ axis and cycle number on the X axis. (A) EHP1. (B) EHP 2. (C) EHP 3. (D) EHP 4. (E) Negative control.

\section{Fig.2, Specificity test of the real-time LAMP for Enterocytozoon hepatopenaei}

Three different pathogen templates were performed along with Enterocytozoon hepatopenaei (EHP). (A) EHP 3. (B) EHP 4. (C) White spot syndrome virus (WSSV). (D) Macrobrachium rosenbergii noda virus (MrNV). (E) Infectious hypodermol and hematopoietic necrosis virus (IHHN). (F) Negative control.

\section{Fig.3, Sensitivity of real-time LAMP for Enterocytozoon hepatopenaei}

The fluorescence unit vs. cycle graph was plotted from the data of the StepOne Real-Time PCR System. Serial 10 -fold dilutions ranging from $10^{0}$ times to $10^{-6}$ times for Enterocytozoon hepatopenaei (EHP) positive sample DNA. (NG) Negative control.

\section{Fig.4, Visual inspection of the LAMP amplicons for Enterocytozoon hepatopenaei} (A) Negative control. (B) Positive control. (C) Enterocytozoon hepatopenaei (EHP). (D-I)

Corresponding to serial 10 -fold dilutions ranging from $10^{0}$ times to $10^{-6}$ times for EHP positive sample DNA, respectively. (J) Negative control. 
283 Fig.5, The amplification efficiency of real-time LAMP compared to the LAMP

284 (A) Real-time Loop-Mediated Isothermal Amplification. (B) Conventional Loop-Mediated

285 Isothermal Amplification. (C) Negative control.

286

287

288

289 
290

291

292

293

294

295

296

297

298

299

300

301

302

303

304

305

306

307

308

309

310

311

312

313

314

315

316

317

318

319

320

\section{References.}

Aldama-Cano DJ, Sanguanrut P, Munkongwongsiri N, Ibarra-Gámez JC, Itsathitphaisarn O, Vanichviriyakit R, Flegel TW, Sritunyalucksana K, and Thitamadee S. 2018. Bioassay for spore polar tube extrusion of shrimp Enterocytozoon hepatopenaei (EHP). Aquaculture 490:156-161. 10.1016/j.aquaculture.2018.02.039

Aranguren LF, Han JE, and Tang KFJ. 2017. Enterocytozoon hepatopenaei (EHP) is a risk factor for acute hepatopancreatic necrosis disease (AHPND) and septic hepatopancreatic necrosis (SHPN) in the Pacific white shrimp Penaeus vannamei. Aquaculture 471:37-42. https://doi.org/10.1016/j.aquaculture.2016.12.038

Chayaburakul K, Nash G, Pratanpipat P, Sriurairatana S, and Withyachumnarnkul B. 2004. Multiple pathogens found in growth-retarded black tiger shrimp Penaeus monodon cultivated in Thailand. Dis Aquat Organ 60:89-96. 10.3354/dao060089

Karthikeyan K, Sharma A, Mekata T, Itami T, and Sudhakaran R. 2017. Rapid and sensitive real-time loop meditated isothermal amplification for the detection of Enterocytozoon hepatopenaei of shrimp. Aquaculture 481:119-123. https://doi.org/10.1016/j.aquaculture.2017.08.036

Lau YL, Meganathan P, Sonaimuthu P, Thiruvengadam G, Nissapatorn V, and Chen Y. 2010. Specific, sensitive, and rapid diagnosis of active toxoplasmosis by a loop-mediated isothermal amplification method using blood samples from patients. J Clin Microbiol 48:3698-3702. 10.1128/jcm.00462-10

Liu YM, Qiu L, Sheng AZ, Wan XY, Cheng DY, and Huang J. 2017. Quantitative detection method of Enterocytozoon hepatopenaei using TaqMan probe real-time PCR. J Invertebr Pathol. 10.1016/j.jip.2017.12.006

Mori Y, Kitao M, Tomita N, and Notomi T. 2004. Real-time turbidimetry of LAMP reaction for quantifying template DNA. Journal of Biochemical and Biophysical Methods 59:145-157. 10.1016/j.jbbm.2003.12.005

Notomi T, Okayama H, Masubuchi H, Yonekawa T, Watanabe K, Amino N, and Hase T. 2000. Loop-mediated isothermal amplification of DNA. Nucleic Acids Research 28. https://doi.org/10.1093/nar/28.12.e63

Rajendran KV, Shivam S, Ezhil Praveena P, Joseph Sahaya Rajan J, Sathish Kumar T, Avunje S, Jagadeesan V, Prasad Babu SVANV, Pande A, Navaneeth Krishnan A, Alavandi SV, and 
Vijayan KK. 2016. Emergence of Enterocytozoon hepatopenaei (EHP) in farmed Penaeus (Litopenaeus) vannamei in India. Aquaculture 454:272-280. 10.1016/j.aquaculture.2015.12.034

Sritunyalucksana K, Srisala J, McColl K, Nielsen L, and Flegel TW. 2006. Comparison of PCR testing methods for white spot syndrome virus (WSSV) infections in penaeid shrimp. Aquaculture 255:95-104. 10.1016/j.aquaculture.2005.12.002

Tang KFJ, Han JE, Aranguren LF, White-Noble B, Schmidt MM, Piamsomboon P, Risdiana E, and Hanggono B. 2016. Dense populations of the microsporidian Enterocytozoon hepatopenaei (EHP) in feces of Penaeus vannamei exhibiting white feces syndrome and pathways of their transmission to healthy shrimp. $J$ Invertebr Pathol 140:1-7. 10.1016/j.jip.2016.08.004

Tang KFJ, Pantoja CR, Redman RM, Han JE, Tran LH, and Lightner DV. 2015. Development of in situ hybridization and PCR assays for the detection of Enterocytozoon hepatopenaei (EHP), a microsporidian parasite infecting penaeid shrimp. Journal of Invertebrate Pathology 130:37-41. https://doi.org/10.1016/j.jip.2015.06.009

Tangprasittipap A, Srisala J, Chouwdee S, Somboon M, Chuchird N, Limsuwan C, Srisuvan T, Flegel TW, and Sritunyalucksana K. 2013. The microsporidian Enterocytozoon hepatopenaei is not the cause of white feces syndrome in whiteleg shrimp Penaeus (Litopenaeus) vannamei. BMC Vet Res 9:139. 10.1186/1746-6148-9-139

Thi Ha N, Dong H, Thanh Thuy N, and Thi Kim Lien V. 2010. Enterocytozoon hepatopenaei has been detected parasitizing tiger shrimp (Penaeus monodon) cultured in Vietnam and showing white feces syndrome (In Vietnamese with English abstract).

Tourtip S, Wongtripop S, Stentiford GD, Bateman KS, Sriurairatana S, Chavadej J, Sritunyalucksana K, and Withyachumnarnkul B. 2009. Enterocytozoon hepatopenaei sp. nov. (Microsporida: Enterocytozoonidae), a parasite of the black tiger shrimp Penaeus monodon (Decapoda: Penaeidae): Fine structure and phylogenetic relationships. J Invertebr Pathol 102:21-29. 10.1016/j.jip.2009.06.004

Cai XQ, Yu HQ, Bai JS, Tang JD, Hu XC, Chen DH, Zhang RL, Chen MX, Ai L, and Zhu XQ. 2012. Development of a TaqMan based real-time PCR assay for detection of Clonorchis sinensis DNA in human stool samples and fishes. Parasitol Int 61:183-186. 10.1016/j.parint.2011.06.010 
Table $\mathbf{1}$ (on next page)

Reference primers used in the study 


Name Sequences $\left(5^{\prime} \rightarrow 3^{\prime}\right) \quad$ Length (nt)

Real-time LAMP

$\begin{array}{lll}\text { EHP-OF AGGTGGGCAAAGAATGAAAT } & 20\end{array}$

$\begin{array}{lll}\text { EHP-OB } & \text { AAGCAGCACAATCCACTC } & 18\end{array}$

EHP-IF CCCAGCATTGTCGGCATAGTATCAAGGACGAAGGCTAGA 39

EHP-IB TGTTGCGAGAGCGATGCTCCTTGCGAGCGTACTATC $\quad 36$

EHP-LF AGAACTACAGCGGTGTCTAATC 22

$\begin{array}{lll}\text { EHP-LB } & \text { TGGTGTGGGAGAAATCTTAGTT }\end{array}$

\section{LAMP}

$\begin{array}{lll}\text { EHP-F3 GGGATCAAGGACGAAGGCT } & 19\end{array}$

$\begin{array}{lll}\text { EHP-B3 GGGATCAAGGACGAAGGCT } & 19\end{array}$

EHP-FIP AAGCATCGCTCTCGCAACACCACACCGCTGTAGTTCTAGCA 41

EHP-BIP TTCGGGCTCTGGGGATAGTACGGTCCTTCCGTCAATTTCGCT 42 
Figure 1

Primers tested for real-time LAMP

The amplification curve graph was plotted automatically by StepOne Real-Time PCR System. $\Delta R n$ is the fluorescence unit minus the baseline, the graph depicts the $\Delta R n$ on the $Y$ axis and cycle number on the X axis. (A) EHP1. (B) EHP 2. (C) EHP 3. (D) EHP 4. (E) Negative control.

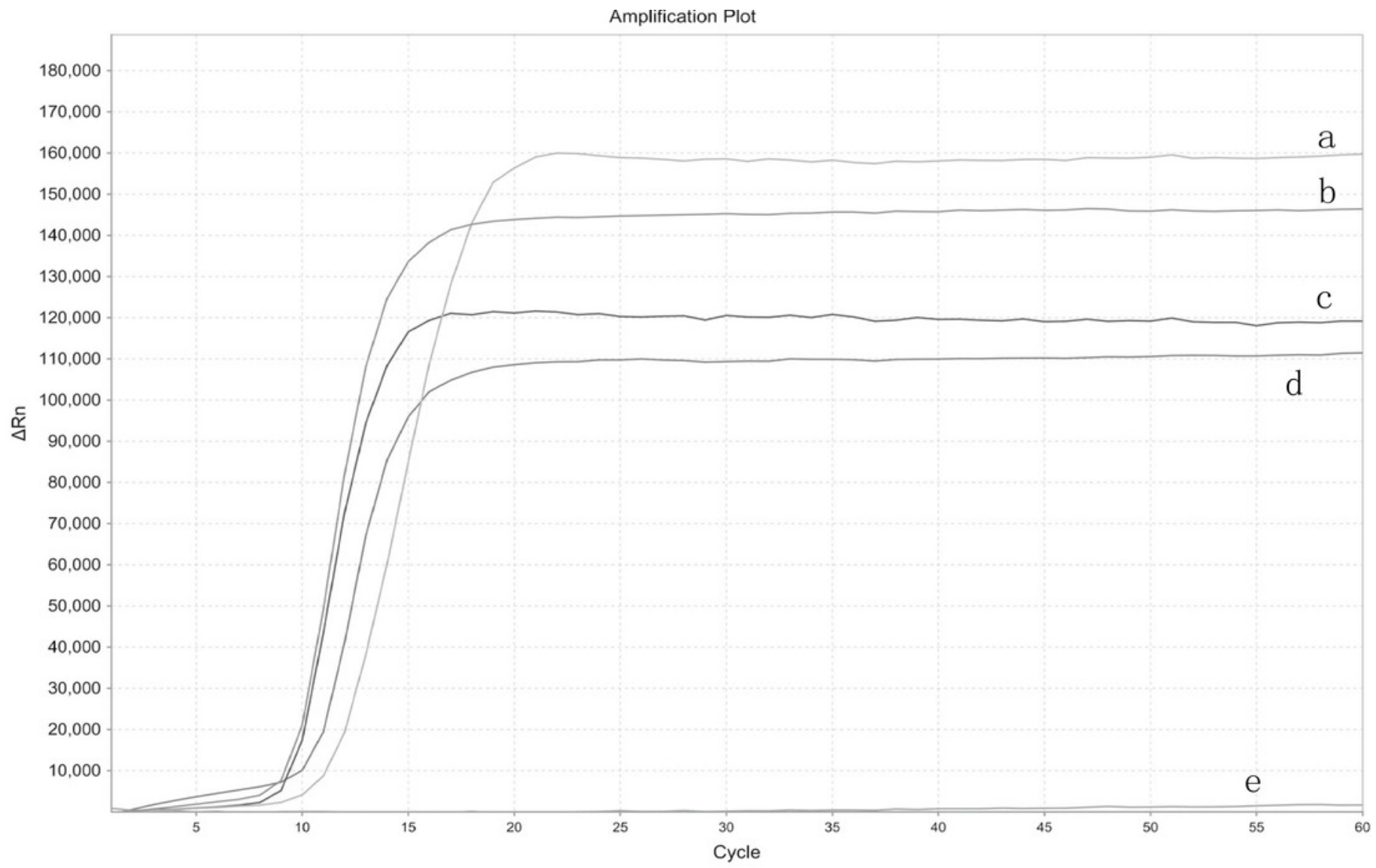


Figure 2

Specificity test of the real-time LAMP for Enterocytozoon hepatopenaei

Three different pathogen templates were performed along with Enterocytozoon hepatopenaei (EHP). (A) EHP 3. (B) EHP 4. (C) White spot syndrome virus (WSSV). (D) Macrobrachium rosenbergii noda virus (MrNV). (E) Infectious hypodermol and hematopoietic necrosis virus (IHHN). (F) Negative control.

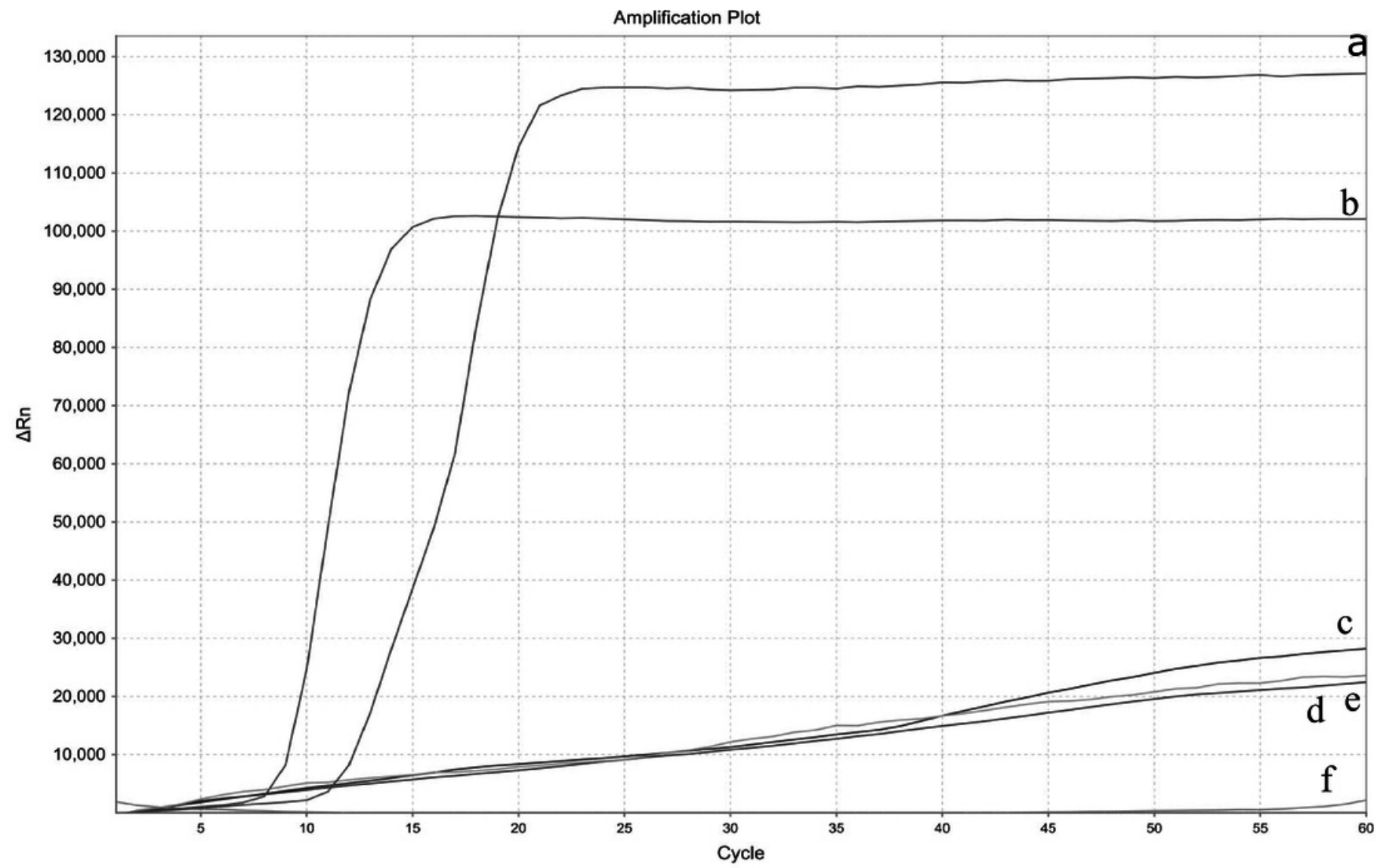


Figure 3

Sensitivity of real-time LAMP for Enterocytozoon hepatopenaei

The fluorescence unit vs. cycle graph was plotted from the data of the StepOne Real-Time PCR System . Serial 10 -fold dilutions ranging from $10^{0}$ times to $10^{-6}$ times for Enterocytozoon hepatopenaei (EHP) positive sample DNA. (NG) Negative control.

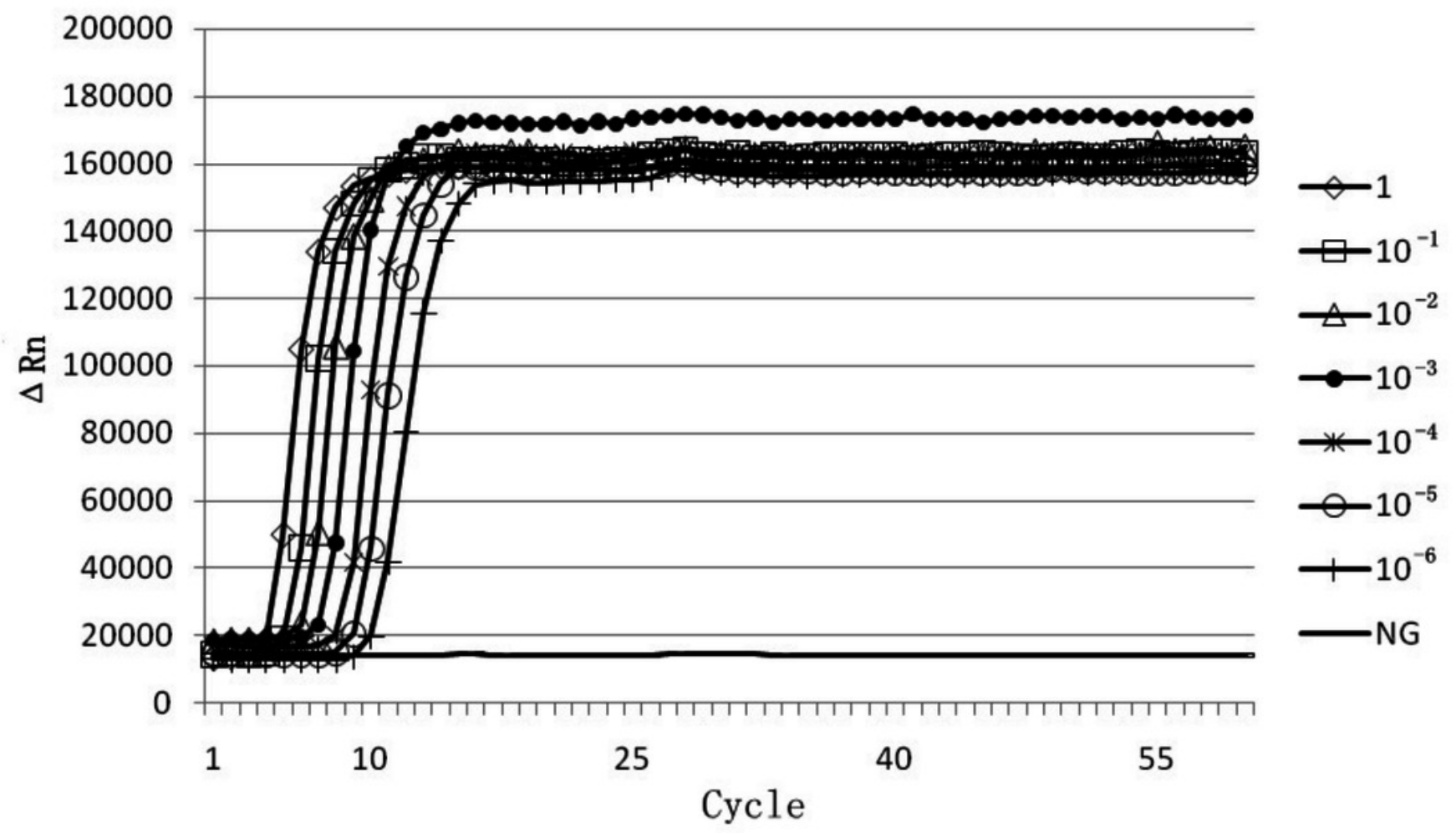




\section{Figure 4}

Fig.4,Visual inspection of the LAMP amplicons for Enterocytozoon hepatopenaei

(A) Negative control. (B) Positive control. (C) Enterocytozoon hepatopenaei (EHP). (D-I)

Corresponding to serial 10 -fold dilutions ranging from $10^{0}$ times to $10^{-6}$ times for EHP positive sample DNA, respectively . (J) Negative control

*Note: Auto Gamma Correction was used for the image. This only affects the reviewing manuscript. See original source image if needed for review.

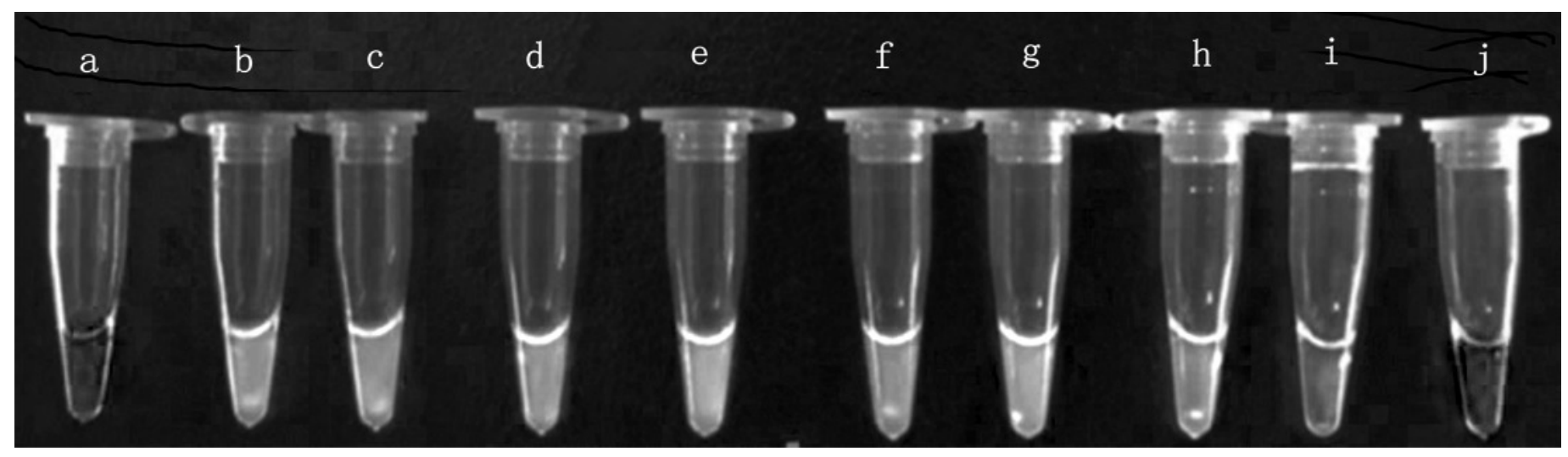


Figure 5

The amplification efficiency of real-time LAMP compared to the LAMP

(A) Real-time Loop-Mediated Isothermal Amplification. (B) Conventional Loop-Mediated Isothermal Amplification. (C) Negative control.

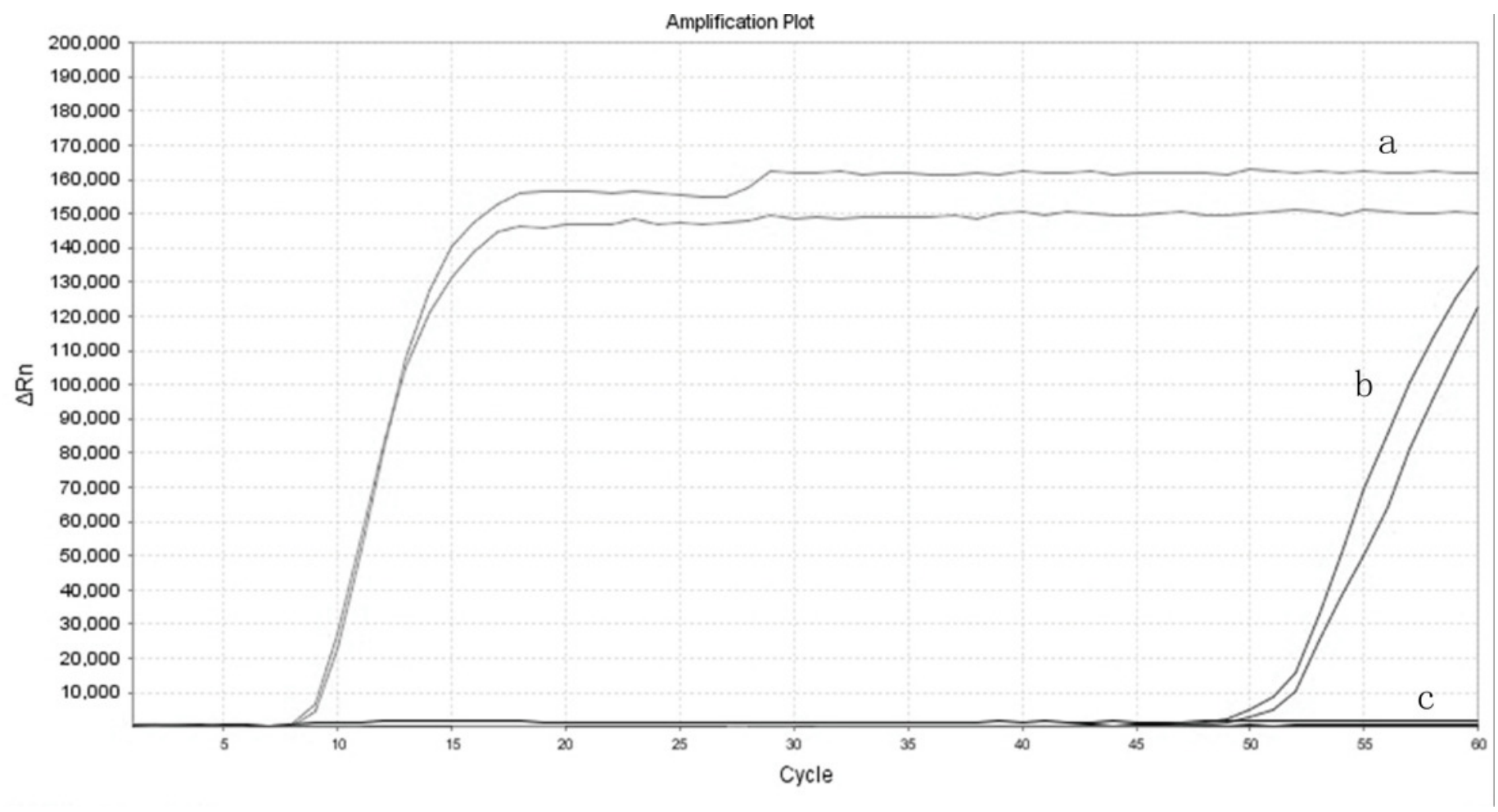

